\title{
A polynomial neural network for river's water-level prediction
}

\author{
Anastasios Rigos $^{1}$ [ - Maria Krommyda ${ }^{1} \cdot$ Athanasia Tsertou $^{1} \cdot$ Angelos Amditis $^{1}$
}

Received: 7 August 2019 / Accepted: 25 February 2020 / Published online: 2 March 2020

(c) Springer Nature Switzerland AG 2020

\begin{abstract}
Flood risk prediction is based on collecting long time-series data using in situ sensors. As such, the consistency and quality of the hydrological flood models is subsequent to information gaps included in the aforementioned time series, which may cause issues that negatively affect the efficiency of the prediction. Herein, to cope with that problem, a novel polynomial neural network is developed, which utilizes Legendre polynomial activation functions and it is able to fill those gaps by predicting the river's water level by intertwining observations coming from different areas of the river, and weather data. The network is trained by a modified evolutionary computation algorithm, which is based on the wellknown artificial bee colony method. The experimental case study concerns the Kifisos river basin (Attica, Greece), where a number of sensors have been deployed to collect data. The network was compared to a feed-forward neural network in terms of the root mean square error performance index. Results indicated a superior performance of the proposed network obtaining predictions from 1 to $2 \mathrm{~cm}$.
\end{abstract}

Keywords Artificial neural network · Polynomial neural network · Water-level prediction · Flood prediction · Legendre Polynomials $\cdot$ Artificial bee colony

\section{Introduction}

Europe has invested a lot infrastructure to achieve an accurate Earth observation capacity. Initiatives such as the Copernicus system provide a mapping of forest areas, wetlands or artificial surfaces [1]; yet, the burden of investing in new equipment or maintaining the current infrastructure is unsustainable [2]. Specifically, flood risk prediction has been traditionally based on models that are developed from time series of data collected over long periods of time from expensive and hard to maintain in situ sensors available only in specific areas [3]. SCENT is an EU-funded project that has created a toolbox of smart collaborative and innovating technologies (Scent Toolbox) that augment costly in situ infrastructure, aiming to enable citizens to monitor Land Cover/Use (LC/LU) changes and how these affect flood phenomena in their urban or rural areas. Experts in the field of flood models define areas of interest and ask volunteers to collect river measurements at these areas. In order to get the river measurements with a consistent and accurate way from volunteers that have no technical knowledge, the volunteers are asked to collect images and videos. A tool has been developed that uses state of the art image recognition techniques in order to extract the water level from images containing a waterlevel indicator and a second one that uses state of the art video processing algorithms in order to extract the water surface velocity from a video containing a tennis ball moving on the surface of a water body. In order to

Electronic supplementary material The online version of this article (https://doi.org/10.1007/s42452-020-2328-9) contains supplementary material, which is available to authorized users.

Anastasios Rigos, anastasios.rigos@iccs.gr; Maria Krommyda, maria.krommyda@iccs.gr; Athanasia Tsertou, athanasia.tsertou@iccs.gr; Angelos Amditis, a.amditis@iccs.gr| ${ }^{1}$ Institute of Communication and Computer Systems (ICCS), National Technical University of Athens, Athens, Greece. 
evaluate the results of these processing tools as well as the flood models created using the crowd-sourced measurements, it is of utmost importance to have traditional information, time series of data collected over long periods.

Regarding this paper, in situ monitoring stations have been installed at the Kifisos pilot area (Attica, Greece) to continuously provide measurements of water level on selected river areas. These stations were installed on carefully selected spots within the river basin based on some requirements. First, it is important to acquire measurements that represent the whole basin, so that the locations are chosen in order to have a fair distribution. Extra focus is given at the output of the basin, the area that lies before the stream goes underground, because it is of major hydrological importance [4]. Furthermore, having such spread across the basin also gives information about the response of different parts of the mainstream segment, along with travel times during major rain events. Second, the sensors are installed to areas of high hydrological interest/needs that comply with a number of characteristics, such as: (a) the distance of the sensor location to any stream turn (both upstream and downstream) is such so it is considered that the turns do not affect the flow within the stream (i.e., the stream is flowing in a straight line for a significant distance both upstream and downstream of the sensor location, ensuring that no biased measurements are taken. (b) No permanent obstacles (e.g., boulders, branches, man-made constructions) in the vicinity of the sensors are present because these kind of obstacles must be avoided to ensure an undisturbed flow toward the point of measurement; (c) The cross-section, at least closer to the output of the basin, is normal. This is required so that the measurements collected by volunteers can be easily comparable.

In order to meet the above-mentioned needs, five water-level sensors were installed at the Kifisos River, at the areas of Kokkinos Milos, Dekeleia, Renti, Monastiri and Varybopi. These sensors use the data connection of a GSM/ GPRS modem working with a SIM card to transmit the collected measurements.

After the installation of the sensors, many issues regarding their maintenance appeared and they led to a significant data loss due to the following issues.

1. Data logger configuration: The sensors collect one measurement every $15 \mathrm{~min}$. These are grouped together in bunches at the data logger and send over GSM network. Finding the optimal bunch size depends on the battery capabilities, the network limitations regarding the size of exchanged data as well as the network quality. Small bunches consume a lot of battery while large batches require good network band- width and quality. During the configuration of the data loggers, bunches of measurements were lost due to network connection errors and restrictions.

2. Battery replacement: Due to the fact that the sensors are installed in different areas, with different network qualities, the battery consumption is not consistent. Sensors at areas with poor network connection have higher battery consumption. As a result, batteries need to be replaced in inconsistent time intervals and occasionally measurements are lost between such replacements.

3. Data reception: The data loggers are pushing the measurements to the back end server. In case of unavailability of the back end server, due to power loss, maintenance or network loss, the measurements are lost.

4. Network loss: The most important problem regarding the loss of measurements is related to the sensor station at the Varybopi area (Fig. 1b). Due to the rural environment, and the topology of the selected area (is installed deep into the woods and in a cliff), the network connectivity is poor. That data logger only sporadically is able to send measurements to the back end server.

Polynomial neural networks (PNNs) consist of a class of artificial neural networks that use high-order functional representations to establish highly non-linear input-output relationships. They are formed by embedding polynomial functions into the network's topology. PNNs have been shown to form effective frameworks in a wide range of applications, such as function approximations [5], channel equalization in wireless networks [6], classification problems [7], image compression [8] and shoreline extraction from video images $[9,10]$.

Many researchers have deployed Artificial Neural Networks (ANNs) in order to simulate or predict river's characteristics such as the water flow. Delafrouz et al. [11] use ANN and a chaos-based artificial neural network model in order to simulate the rivers flow calculated as the ratio of the water volume over time. Campolo et al. [12] forecast river-floods with a feed-forward neural network using as input parameters the river's water level on previous timestamps (the water level 1, 2, 3 and 4 hours before) and rainfall data. Zhu et al. [13] model the daily temperature of a river using an ANN and a neuro-fuzzy system using as input parameters the air temperature, the river flow discharge and the calendar components (the day, the month and the year). Yaseen et al. [14] compare feed-forward neural network (FFNN) and radial basis function neural networks (RBFNN) to forecast the daily streamflow of river; an input of the ANN's the use the river's streamflow of previous days. Kasiviswanathan et al. [15] use ANN's and 
Fig. 1 a A water-level sensor together with a data logger, installed at the Renti area, $\mathbf{b}$ water-level sensors (yellow marks) and weather stations (blue mark) location. Image background source: Google Earth

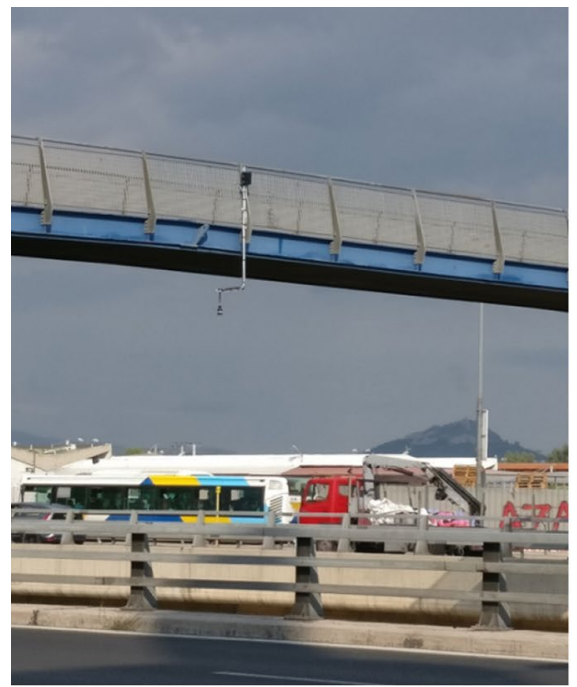

(a)

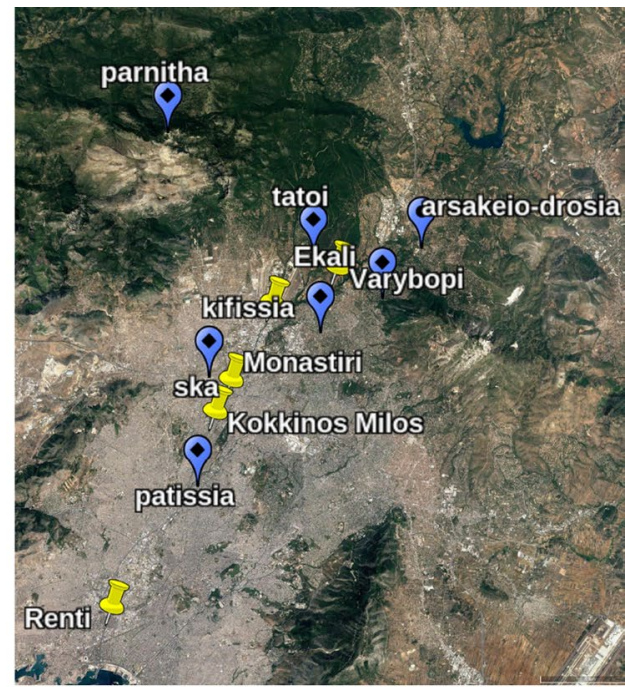

(b) wavelet-based neural networks in order to forecast a river's streamflow and predict floods based on streamflow data of previous days. Khan et al. [16] use fuzzy NN's in order to predict river flood events using as input parameters the daily peak and mean flowrates and some weather conditions related to characteristics of the air.

\subsection{Contribution}

The current contribution recognizes how important is the development of consistent and accurate flood models based on continuous time series, without missing data. As such, a method is developed that can predict the water level in one of the above-mentioned 5 areas by using the data of the other 4. The proposed approach is tested in the following cases: (a) Completing the time series collected so far from the in situ sensor that have gaps created due to maintenance issues; (b) Complete time series as received, on real time, if no-data are received; (c) Completely restore time series, for extended time frames, when in situ sensors have serious hardware failures or need to be uninstalled for maintenance reasons; (d) Create an early alert mechanism to inform the system administrators about potential flood hazards; (e) Predict and produce alerts in cases of extreme events, such as flood and drought.

\section{Experimental setup and data acquisition}

Five water-level sensors were installed at the Kifisos River (Fig. 1a), at the areas of Kokkinos Milos, Dekeleia, Renti, Monastiri and Varybopi (Fig. 1b). The equipment used for the in situ sensors is Pulsar dBi transducers (Renti, Kokkinos Mylos), Adcon LEV1 level sensors (Dekeleia, Monastiri and Varybopi) and Summerton Stylitis-20 data loggers. The special features that each of the chosen locations (e.g., accessibility to the stream, installation on a bridge and cross-section geometry) have made it so that either a pressure transducer (Adcon LEV1 Level Sensor) or an ultrasonic measurement transducer (Pulsar dBi transducer) was a better option and had to be used. The data loggers work with lithium batteries, they were set to record data every $15 \mathrm{~min}$, they store those data to the internal $4 \mathrm{MB}$ flash memory and they transmit the data once per day. For the data transmission, they use the data connection of a GSM/ GPRS modem working with a SIM card and the acquired data are sent to a predefined FTP file server maintained by the authors.

For the water-level's prediction, data from ground weather stations were used together will the water-level time series. In order to simulate the above regression problem, an artificial neural network (ANN) was used.

The collected water-level time series consist of measurements from 2017/09/28 up to now (2019/6/25) with frequency of 1 measurement per $15 \mathrm{~min}$. The water level of the river depicts the water collected in the river due to rainfalls on nearby areas, so rainfall data will be used as extra indicators. Rainfall data (daily data, measured in $\mathrm{mm}$ ) were collected from the archive of the National Observatory of Athens (NOA) using their open-access online database at the website http://meteosearch.meteo.gr/. The weather stations of NOA that are close to the river are the ones at the areas: arsakeio-drosia, ekali, kifissia, parnitha, patissia, ska, tatoi and their location is presented in Fig. $1 \mathrm{~b}$. Unfortunately, hourly rainfall data were not freely available online by NOA, so only the daily are used.

To create a regression dataset from water level and rainfall data, the data of 1 year's time series with observations 
(2017/09/28 to 2018/09/27) were used from both datasets. From the water level, only one measurement per $6 \mathrm{~h}$ was used and each of these measurements was associated with the rainfall of this day. The form of these data is presented on Fig. 2.

Our database should contain 1460 measurements (4 measurements per day for 365 days) but due to missing data of water level or rainfall, the dataset consists of $N=665$ data of the form:

Data $=\left[w_{K}, w_{D}, w_{R}, w_{M}, w_{V}, r_{a}, r_{e}, r_{k}, r_{\text {par }}, r_{\text {pat }}, r_{s}, r_{t}\right]$

where the first 5 parameters are the water-level data of the 5 areas and the next ones are the daily rainfall measurements of the 7 weather stations.

As already mentioned in the introduction, the most challenging sensor to collect the measurements is the one installed at the Varybopi area. So here the main goal is to predict the water level at the Varybopi area, so the dataset was transformed as:

$$
S=\left\{\left(\mathbf{x}_{k}, y_{k}\right): \mathbf{x}_{k}=\left[x_{k 1}, x_{k 2}, \ldots, x_{k p}\right], \quad k=1,2, \ldots, N\right\}
$$

where the input data $\mathbf{x}$ are presented as a $p$-dimensional vector presenting the water-level data of the 4 areas (Varybopi was excluded) and the rainfall data of the 7 areas, so $p=11$ in here. The output for the regression ANN (i.e., the data the ANN will predict) will be the water level of Varybopi area, so $y=w_{v}$. Those data can be found online using the link http://scent-harm.iccs.gr/NNdata.csv.

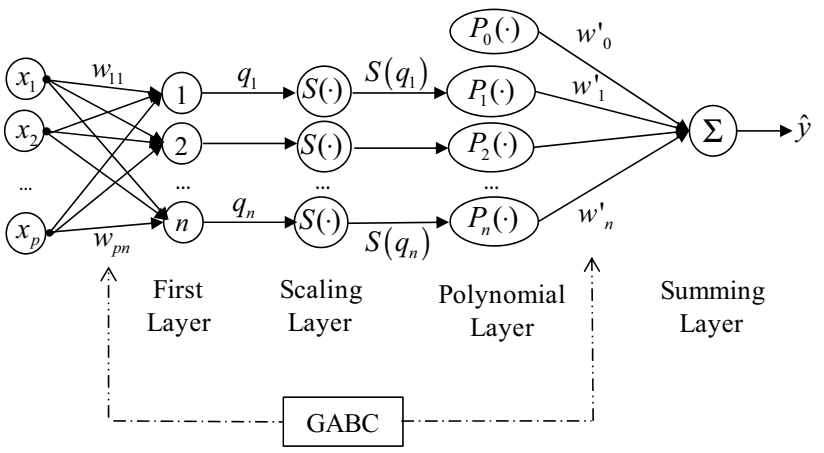

Fig. 3 The topology of the LPNN

\section{The proposed artificial neural network}

\subsection{The legendre polynomial neural network (LPNN)}

The neural network that will be used in here has similar structure to the one presented by Rigos et al. in [17], but a total different training process. It is a polynomial neural network using Legendre polynomials as activation functions in its nodes. It will be noted as LPNN in here. The Legendre polynomials can be defined by the recurrence relation which is called Bonnet's recursion formula $[18,19]$ :

$$
\left\{\begin{array}{ll}
P_{0}(x)=1 & P_{1}(x)=x \\
P_{n}(x)=\frac{1}{n}\left[(2 n-1) x P_{n-1}(x)-(n-1) P_{n-2}(x)\right] & n \geq 2
\end{array}\right\}
$$

Those are orthogonal in the interval $[-1,1]$, satisfying the inner-product equation:

$$
\left\langle P_{m}(x), P_{n}(x)\right\rangle=\int_{-1}^{1} P_{m}(x) P_{n}(x) \mathrm{d} x=\frac{2}{2 n+1} \delta_{m n}=\left\{\begin{array}{cc}
\frac{2}{2 n+1}, & m=n \\
0, & m \neq n
\end{array}\right.
$$

Fig. 2 a The water-level data collected by the sensors and b the daily rainfall collected from NOA

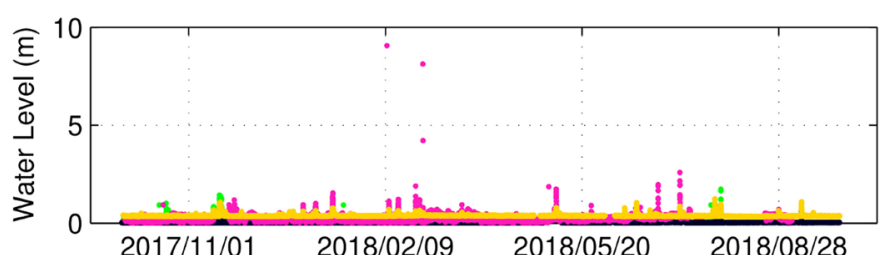

(a)

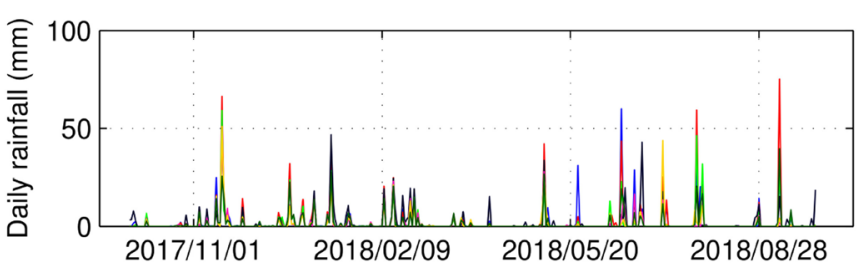

(b)
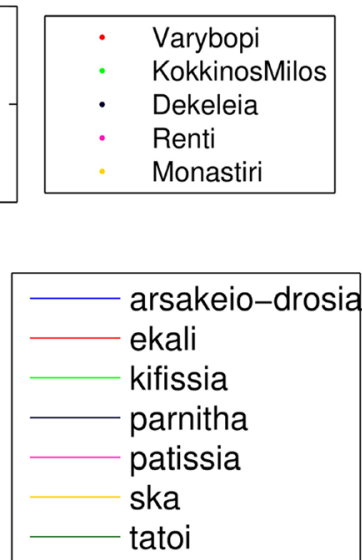

SN Applied Sciences 
where $\delta_{m n}$ is the Kronecker delta function. The structure of the LPNN that will be used is presented on Fig. 3.

Using a dataset with the form described in Eq. (2), the analysis of the LPNN follows. For a given input $\mathbf{x}$ of the form $\mathbf{x}=\left[x_{1}, x_{2}, \ldots, x_{p}\right]$, the first layer of the LPNN creates a linear combination of the input variables:

$q_{\ell}=\sum_{j=1}^{p} x_{j} w_{j \ell}, \quad j=1,2, \ldots, p, \quad \ell=1,2, \ldots, n$

with $w_{j \ell} \in \mathbb{R}$ the weighting parameters. Since each of the $q_{\ell}$ does not necessary belong in the $[-1,1]$ interval that Legendre polynomials operate [Eq. (4)], in the second layer (called Scaling layer) the data are scaled. The scaling process follows. After calculating the minimum and maximum values of the input data in each dimension $j$, each of input parameters belongs in the interval: $x_{j} \in\left[\min _{k=1,2 \ldots, N}\left(x_{k j}\right), \max _{k=1,2 \ldots, N}\left(x_{k j}\right)\right]=\left[x_{j}^{\mathrm{MIN}}, x_{j}^{\mathrm{MAX}}\right]$. Also, for each of the weights $w_{j \ell}$ we have that: $w_{j \ell} \in\left[w_{L}, w_{U}\right]$ where the parameters $w_{L}, w_{U}$ should be manually chosen before the training process of the LPNN and should not change during the whole training process. Each of the $x_{j} w_{j \ell}$ products of Eq. (5) belongs in the interval $m_{j} \leq w_{j \ell} x_{j} \leq M_{j}, \forall \ell=1,2, \ldots, n, \forall j=1,2, \ldots, p$, where:

$m_{j}=\min \left\{w_{L} x_{j}^{\mathrm{MIN}}, w_{L} x_{j}^{\mathrm{MAX}}, w_{U} x_{j}^{\mathrm{MIN}}, w_{U} x_{j}^{\mathrm{MAX}}\right\}$

$M_{j}=\max \left\{w_{L} x_{j}^{\mathrm{MIN}}, w_{L} x_{j}^{\mathrm{MAX}}, w_{U} x_{j}^{\mathrm{MIN}}, w_{U} x_{j}^{\mathrm{MAX}}\right\}$

so summing a total of $p$ products of the form: $x_{j} w_{j \ell}$ we have that

$\sum_{j=1}^{p} m_{j} \leq \sum_{j=1}^{p} x_{j} w_{j \ell} \leq \sum_{j=1}^{p} M_{j} \Rightarrow q^{\mathrm{MIN}} \leq q_{\ell} \leq q^{\mathrm{MAX}}$

In order to scale the data $q_{\ell} \in\left[q^{\mathrm{MIN}}, q^{\mathrm{MAX}}\right]$ into the data $S\left(q_{\ell}\right) \in[-1,1]$ the linear scaling transformation will be used [20]:

$S\left(q_{\ell}\right)=-1+2 \frac{q_{\ell}-q^{\mathrm{MIN}}}{q^{\mathrm{MAX}}-q^{\mathrm{MIN}}}$

At the 3rd layer (the Polynomial Layer) there are $n+1$ nodes that use the Legendre polynomials of degrees 0 to $n$ as activation functions. So the output of the Polynomial Layer is

$P_{\ell}=P_{\ell}\left(S\left(q_{\ell}\right)\right), \quad \ell=1,2, \ldots, n$ and for $\ell=0$ the bias of the network is $P_{0}=1$. Summing those with the use of the weights $w_{\ell^{\prime}}^{\prime} 0 \leq \ell \leq n$ the network's output is as follows:

$\hat{y}=\sum_{\ell=0}^{n} w_{\ell}^{\prime} P_{\ell}\left(S\left(q_{\ell}\right)\right)$

which can be written as

$\hat{y}=w_{0}^{\prime}+w_{1}^{\prime} P_{1}\left(S\left(q_{1}\right)\right)+\cdots+w_{n}^{\prime} P_{n}\left(S\left(q_{n}\right)\right)$

Based on Eq. (12) it has to be noted that the networks output $\hat{y}$ is not a polynomial-series expansion, it would be one only if $S\left(q_{1}\right)=S\left(q_{2}\right)=\cdots=S\left(q_{n}\right)$.

\subsection{Training of the LPNN}

The training of the LPNN is the procedure of estimating the values of the weights $w_{j \ell}, \forall \ell=1,2, \ldots, n, \forall j=1,2, \ldots, p$ and $w_{\ell}^{\prime}, \ell=0,1, \ldots, n$ in order to minimize the RMSE of the LPNN:

RMSE $=\sqrt{\frac{1}{N} \sum_{k=1}^{N}\left(y_{k}-\hat{y}_{k}\right)^{2}}$

subject to $w_{j \ell} \in\left[w_{L}, w_{U}\right]$. In this optimization problem, the estimation of parameters $w_{j \ell}$ is a constrained optimization problem and the estimations of $w_{\ell}^{\prime}$ is an unconstrained one.

In order to solve this optimization problem, a modification of the artificial bee colony (ABC) [21] algorithm is used. This modification is inspired from particle swarm optimization (PSO) algorithm of Kennedy and Eberhart [22] and applies the global best ( $\mathbf{g}^{\text {best }}$ ) feature of the PSO in the $A B C$ algorithm, it will be called GABC and is originally proposed by Zhu and Kwong [23]. One small modification was applied to the GABC concerning of the unconstrained optimization part. The analysis of the GABC follows.

\subsection{The GABC algorithm}

According to Karaboga $[21,24]$ the goal of the $A B C$ is to find the point $\mathbf{v}^{*}=\max _{x \in \Theta}($ Fitness $(\mathbf{v}))$ where:

$\operatorname{Fitness}(\mathbf{v})= \begin{cases}\frac{1}{1+\operatorname{RMSE(v)})^{\prime}}, & \text { if } \operatorname{RMSE}(\mathbf{v}) \geq 0 \\ 1+|\operatorname{RMSE}(\mathbf{v})|, & \text { if } \operatorname{RMSE}(\mathbf{v})<0\end{cases}$

The last equation modifies the minimization problem for the RMSE of the LPNN to a maximization problem. For 
the RMSE optimization problem, only the first sub-function of the Eq. (14) is used, since RMSE is always a non-negative number. The solutions' search space $\Theta$ is bounded as: where $k$ is randomly chosen from the set: $\{1,2, \ldots, r\}-i$. In the $\mathrm{GABC}$ algorithm, the above equation becomes:

$\Theta=\left\{\mathbf{v}=\left[v_{1}, v_{2}, \ldots, v_{r}\right] \in \mathbb{R}^{r} / v_{\lambda}^{\min } \leq v_{\lambda} \leq v_{\lambda}^{\max }, \quad \forall \lambda=1,2, \ldots, r\right\}$

For the proposed LPNN training problem, the solution that the GABC algorithm searches is the vector containing all the training parameters (weights) of the LPNN:

$\mathbf{v}=\left[w_{11}, w_{12}, \ldots, w_{p n}, w_{0}^{\prime}, w_{1}^{\prime}, \ldots, w_{n}^{\prime}\right]^{\top}$

While in the GABC algorithm in [17] the search space is bounded, in here we apply check for the boundaries only for the first $p \cdot n$ parameters (for the weights $w_{j \ell}$ it has to be checked that: $w_{L} \leq w_{j \ell} \leq w_{U}$ ) while for the next $n+1$ parameters (weights $w_{t}^{\prime}$ ), no boundary check is applied.

In $A B C$ algorithm, a colony of (artificial) honey-bees search for rich food sources (good solutions to an optimization problem). After initiating some random food source, the bee colony optimizes these sources by (a) moving to better nearby solutions and (b) abandoning not-rich food sources. The algorithm is described in Table 1.

Initial Step: The number SN defines the number solutions (food sources) and CS $=2 \cdot \mathrm{FS}$ is the colony size of the bees. At the initial step of the algorithm, food sources are produced by randomly choosing a total of $\mathrm{SN}$ initial solutions $\mathbf{v}_{i}, i=1,2, \ldots$, SN belonging into space $\Theta$, as:

$v_{i \lambda}=v_{\lambda}^{\min }+\operatorname{rand}(0,1)\left(v_{\lambda}^{\max }-v_{\lambda}^{\min }\right)$

with rand $(0,1)$ a random number in $(0,1)$ and $v_{\lambda}^{\min }, v_{\lambda}^{\max }$ the limits defined in Eq. (15). The population of the bees is split into 2 equal groups, the group of the employed bees and the group of the onlooker bees.

Employed Bees Phase: In the employed bees phase, each employed bee generates a new candidate solution $\mathbf{v}_{i}^{\text {new }}$ by changing its $\lambda$-th component as [18]:

$v_{i \lambda}^{\text {new }}=v_{i \lambda}+\operatorname{rand}(-1,1)\left(v_{i \lambda}-v_{k \lambda}\right)$ $v_{i \lambda}^{\text {new }}=v_{i \lambda}+\operatorname{rand}(-1,1)\left(v_{i \lambda}-v_{k \lambda}\right)+\psi\left(g_{i}^{b}-v_{i \lambda}\right)$

where $g_{i}^{\text {best }}$ is the $i$-th component of the global-best solution that has ever discovered by the whole colony during the algorithm, rand $(-1,1)$ is a random number in $(-1,1)$ and $\psi$ a constant real number. Once the new candidate solution $\mathbf{v}_{i}^{\text {new }}$ is generated, a greedy selection is used. If the fitness value of $v_{i}^{\text {new }}$ is better than that of its previous one $\left(\mathbf{v}_{i}\right)$, then it is updated as $\mathbf{v}_{i} \leftarrow \mathbf{v}_{i}^{\text {new }}$, otherwise $\mathbf{v}_{i}$ remains unchanged.

Onlooker Bees Phase: After all the employed bees complete the search process, they share the information of their food sources with the onlooker bees through waggle dances. An onlooker bee evaluates the nectar information taken from all employed bees and chooses a food source with a probability related to its nectar amount. This probabilistic selection is really a roulette wheel selection mechanism which is described as equation below:

$P_{i}=\frac{\text { Fitness }_{i}}{\sum_{i=1}^{\mathrm{SN}} \text { Fitness }_{i}}$

where Fitness $s_{i}$ is the fitness value of the $i$-th solution in the swarm.

Scout Bees Phase: If a position cannot be improved over a predefined number of cycles (called limit, equal to $L=\mathrm{SN} \cdot r$, where $r$ is the dimension of the search space), then this food source is abandoned. The abandoned position is re-generated with the equation of the initial step [Eq. (17)].

Table 1 The GABC algorithm

- Initial Step: Initial food sources are produced for all employed bees REPEAT

- Employed Bees Phase: Each employed bee goes to a nearby food source and determines if it has a greater nectar amount that the older. If yes, it dances to inform the rest of the bees

- Onlooker Bees Phase: Each onlooker watches the dance of all the employed bees and chooses one of their sources depending on the dances. Then it moves to that source

- Scout Bees Phase: Abandoned food sources are determined and are replaced with the new food sources discovered by scouts

- The best food source (global best) discovered so far is stored

UNTIL a termination criterion is satisfied 


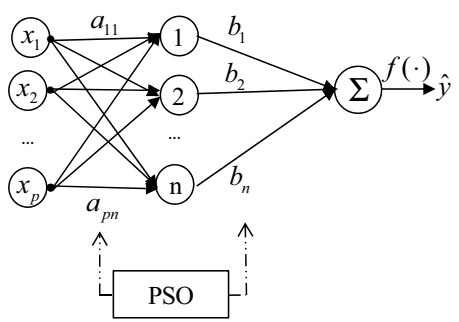

Fig. 4 The topology of a simple FFNN that was used for comparison reasons

\subsection{A feed-forward neural network for comparison purposes}

For comparison reasons, one more neural network was used. A classic feed-forward neural network (FFNN) with topology as presented in Fig. 4. This network generates the output:

$\widehat{y}=f\left(\sum_{\ell=1}^{n} b_{\ell} a_{\ell}\right)$

where $f(x)=\tanh \left(\frac{x}{2}\right)$ is the activation function and $q_{\ell}=\sum_{j=1}^{p} x_{j} a_{j \ell}, \ell=1,2, \ldots, n$. In order to train it, i.e., estimate the $a_{j \ell}$ and $b_{\ell}$ synaptic weights, the PSO algorithm was used.

\section{Results}

In the simulations of the $\mathrm{GABC}$ algorithm, as a colony size the $C S=50$ was used and the $\psi$ of Eq. (19) was set to 1 . As an ending criterion for the GABC, a maximum of 100 iterations was used. For the simulation study, the 10 cross-fold validation method was used and for each of these folds 5 initializations were deployed. While running each of the tenfolds, the $90 \%$ of the dataset was used as training set for the LPNN and the rest $10 \%$ as testing set. The results of these networks are measured with the resulting RMSEs and Table 2 presents these RMSEs in the form of (mean value) \pm (standard deviation). Together with the RMSEs, the mean absolute error (MAE) and the coefficient of determination $R^{2}$ are also presented. The MAE is defined as:

$\mathrm{MAE}=\frac{1}{N} \sum_{k=1}^{N}\left|y_{k}-\hat{y}_{k}\right|$

and the coefficient of determination $R^{2}$ as [25]:

$R^{2}=\left(\frac{\frac{1}{N} \sum_{k=1}^{N}\left(y_{k}-\bar{y}\right)\left(\hat{y}_{k}-\overline{\hat{y}}\right)}{\sqrt{\frac{1}{N} \sum_{k=1}^{N}\left(y_{k}-\bar{y}\right)^{2}} \sqrt{\frac{1}{N} \sum_{k=1}^{N}\left(\hat{y}_{k}-\bar{y}\right)^{2}}}\right)^{2}$

where $\bar{y}$ and $\bar{y}$ present the mean values of $y$ and $\hat{y}$. The results of these metrics are presented in Table 3. The mean values of the RMSEs are presented at Fig. 5. The LPNN was trained with $n=3,4,5$ as Legendre polynomial degrees and for comparison reasons, the FFNN used $n=3,4,5$ hidden layers.

\section{Discussion}

As is presented in Tables 2 and 3 and in Fig. 5, the LPNN outperforms the FFNN for all polynomial degrees. The trend for both networks is to provide better results for greater number of hidden nodes. The values of MAE are similar to the ones of RMSE as it was expected. The results
Table 2 The RMSE of the simulation results

\begin{tabular}{|c|c|c|c|c|c|c|c|c|}
\hline \multirow[t]{3}{*}{$n$} & \multicolumn{4}{|c|}{ MAE in meters (mean) } & \multicolumn{4}{|c|}{$R^{2}$ (mean) } \\
\hline & \multicolumn{2}{|c|}{ Training data } & \multicolumn{2}{|c|}{ Testing data } & \multicolumn{2}{|c|}{ Training data } & \multicolumn{2}{|c|}{ Testing data } \\
\hline & LPNN & FFNN & LPNN & FFNN & LPNN & FFNN & LPNN & FFNN \\
\hline 3 & 0.0259 & 0.1509 & 0.0352 & 0.2486 & 0.3100 & 0.2441 & 0.2088 & 0.1639 \\
\hline 4 & 0.0254 & 0.0513 & 0.0248 & 0.1307 & 0.3079 & 0.3035 & 0.2150 & 0.1837 \\
\hline 5 & 0.0232 & 0.0323 & 0.0185 & 0.0434 & 0.3116 & 0.3106 & 0.2179 & 0.1631 \\
\hline
\end{tabular}

Table 3 The MAE's and $R^{2}$ metrics of the simulation results

\begin{tabular}{|c|c|c|c|c|}
\hline \multirow[t]{3}{*}{$n$} & \multicolumn{4}{|c|}{ RMSEs in meters (mean \pm standard deviation) } \\
\hline & \multicolumn{2}{|l|}{ Training data } & \multicolumn{2}{|l|}{ Testing data } \\
\hline & LPNN & FFNN & LPNN & FFNN \\
\hline 3 & $0.0277 \pm 0.0019$ & $0.2479 \pm 0.3544$ & $0.0216 \pm 0.0062$ & $0.2909 \pm 0.5206$ \\
\hline 4 & $0.0265 \pm 0.0015$ & $0.0805 \pm 0.0536$ & $0.0169 \pm 0.0050$ & $0.0965 \pm 0.1430$ \\
\hline 5 & $0.0248 \pm 0.0001$ & $0.0378 \pm 0.0152$ & $0.0095 \pm 0.0001$ & $0.0411 \pm 0.0221$ \\
\hline
\end{tabular}


Fig. 5 The mean training and testing errors of the neural networks
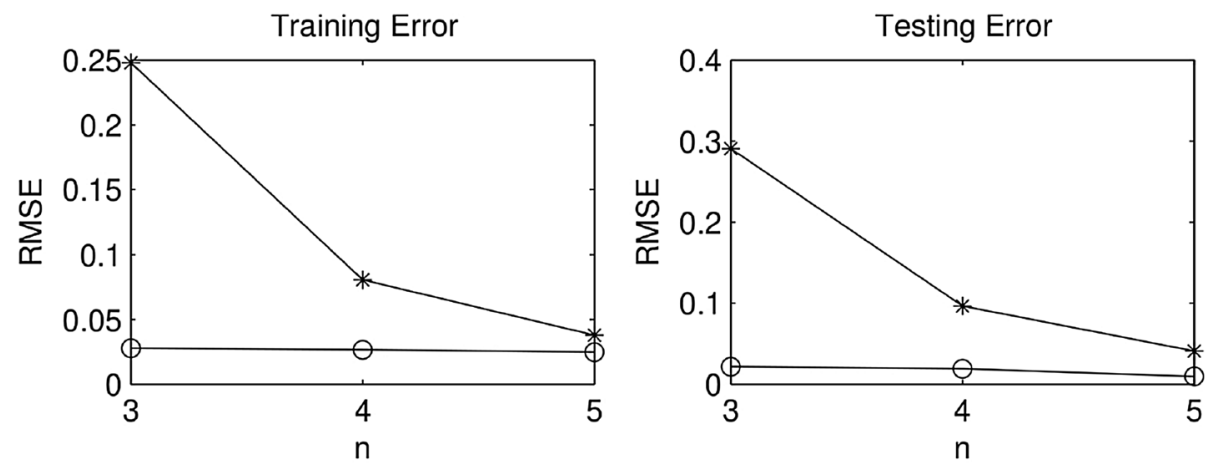

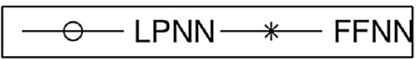

of the $R^{2}$ for the LPNN are about 0.31 for the training data and 0.21 for the testing; the reason this is not so close to 1 is due to the dataset which consists of a high non-linear system. These values should not be compared with values of $R^{2}$ of papers using data hiding linear or other format of relations between the input and output data, since the dataset used in this document is a novel dataset produced by random environmental conditions. In general, RMS errors with values of about $1 \mathrm{~cm}$ such the ones that resulted by the LPNN ( $n=5$, testing process) are considered to be a very good approximation for the water level of a river and can provide a great tool in order to create a flood or extreme rain prediction system. Nevertheless, despite of the low values of the coefficient of determination $\left(R^{2}\right)$, having a system that approximates the water level of a river's flow with a mean error of $1 \mathrm{~cm}$, is considered as a fair enough approximator for the purposes of this document.

While in many studies, such as, e.g., in $[12,14,15]$, only the past time series are used in order to forecast the water level of rivers, and some others use the weather data of the past days together with the time series (e.g., in [16]), the present study follows a completely different approach. The present study uses data provided by sensors installed on areas of the river, together with weather data which can be provided in real-time by national organizations; this methodology was followed because the authors believe that the rainfall in areas near the river is a parameter easy to retrieve, is of crucial importance on the resulting water level, but other studies usually do not use it. Although the authors know that using deep neural networks, such as the ones in $[26,27]$, could improve the network's results, this was avoided, since the authors' demand for small computational complexity on the testing process. By keeping the computational complexity low, the proposed methodology can be applied even on embedded systems or microcontroller units installed together with the sensors (see next section).

\section{SN Applied Sciences}

The presented results, according to the resulting RMSE's, seem to produce smaller error values compared with works solving problems similar to the presented one (e.g., in [28] and [29] the used ANN's result RMSE values of about 0.44 and $0.15-0.36 \mathrm{~m}$ respectively), but those comparisons cannot be absolute, since the used datasets are totally different. In general, in problems as the presented one, each work focuses to a specific scope of the use of ANN's, e.g., in [29] it is mentioned that "the predictions accurately reproduced the rising and falling tendencies of water levels over time" and in [12] the authors focus in the forecasting of the water level, using previous waterlevel measurements; while in this work the authors focus on deploying a fast and accurate regression model. The ANN's architecture of the presented study is not limited on the format of the used data, but it can be applied to similar datasets for the water-level predictions and general on other regression problems.

\section{Conclusion}

In this work, an ANN has been built in order to solve the problem of predicting a river's water level. ANN's can provide an immediate and real-time prediction in such problems, in contrast with hydraulic flood models that need complex setup and calculations [3]. The use of such forecasting systems, become of an important need since the rise of the frequency of extreme weather phenomenal [30]. A regression ANN as the one presented, which is able to provide the water level on a river's area with only $1 \mathrm{~cm}$ error provides an efficient mechanism to predict and warn about extreme events (as, e.g., potential flood hazards) and can result a tool for services as, e.g., the civil protection. As is presented in this work, the use of orthogonal polynomials in the nodes of an ANN combined with the use of swarm intelligence algorithms as the network's optimization method, increases the network's accuracy compared with other networks with similar topologies (the FFNN). 
Is also has to be mentioned that more complex network topologies as FFNN's with many series of hidden nodes (i.e., deep neural networks) were intentionally avoided by the authors in order to present an easy re-producible network with low-demanding of computational complexity.

\section{Future work}

In the future, a live prediction system using the LPNN will be build. This alert system will consist of an algorithm that runs once every day (when the new data from sensors arrive to the FTP server) and applies the LPNN's testing process. This system will produce alerts if the predicted water level at the Varybopi area is expected to reach a specific threshold value. If this threshold is reached, there is a great possibility of flood phenomena or (in general) extreme rain phenomena in the area of Attica. In order to run the testing process of the LPNN only the weight parameters of a network with good testing performance have to be stored. Because of the small complexity of the proposed LPNN, this alert system can also be built into embedded devices, such as, e.g., smartphones or microcontrollers. Also, the system can be built with sensors that can transmit more frequent the collected data, such as, e.g., at $1 \mathrm{~Hz}$. Using more frequent live data from the sensors and using live weather data, a more immediate and accurate early warning alert system using ANN's can be build.

Acknowledgements This research has been financed by European Union's Horizon 2020 research and innovation programme under Grant Agreement No 688930, project SCENT (Smart Toolbox for Engaging Citizens into a People-Centric Observation Web). For more information about the SCENT project visit the website https://scent -project.eu/.

\section{Compliance with ethical standards}

Conflict of interest The authors declare that they have no conflict of interest.

\section{References}

1. Butler D (2014) Earth observation enters next phase. Nat News 508(7495):160

2. Copernicus-The EU's Earth observation and monitoring programme (2017) Think Tank pages of the European Parliament. http://www.europarl.europa.eu/thinktank/en/docum ent.html?reference=EPRS_BRI(2017)608787. Accessed 1 Aug 2019

3. Sorooshian S, Hsu K-L, Coppola E, Tomassetti B, Verdecchia M, Visconti G (2008) Hydrological modelling and the water cycle: coupling the atmospheric and hydrological models, vol 63. Springer, Berlin
4. Remesan R, Mathew J (2016) Hydrological data driven modelling. Springer, Berlin

5. Lee T-T (1998) Jeng J-T (1998) The Chebyshev-polynomialsbased unified model neural networks for function approximation. IEEE Trans Syst Man Cybern Part B (Cybern) 28(6):925-935

6. Patra JC, Meher PK, Chakraborty G (2009) Nonlinear channel equalization for wireless communication systems using Legendre neural networks. Signal Process 89(11):2251-2262

7. Naik B, Nayak J, Behera HS, Abraham A (2016) A self adaptive harmony search based functional link higher order ANN for non-linear data classification. Neurocomputing 179:69-87

8. Ma L, Khorasani K (2000) Adaptive structure feed-forward neural networks using polynomial activation functions. In: International society for optics and photonics, pp 120-129

9. Rigos A, Tsekouras GE, Vousdoukas MI, Chatzipavlis A, Velegrakis AF (2016) A Chebyshev polynomial radial basis function neural network for automated shoreline extraction from coastal imagery. Integr Comput Aided Eng 23(2):141-160

10. Rigos A, Vousdoukas MI, Andreadis OP, Tsekouras GE, Velegrakis $A F$ (2014) On the systematic implementation of artificial neural networks in the classification of variance images and shoreline extraction. Fresenius Environ Bull 23(11):2677-2686

11. Delafrouz H, Ghaheri A, Ghorbani MA (2018) A novel hybrid neural network based on phase space reconstruction technique for daily river flow prediction. Soft Comput 22(7):22052215. https://doi.org/10.1007/s00500-016-2480-8

12. Campolo M, Andreussi P, Soldati A (1999) River flood forecasting with a neural network model. Water Resour Res 35(4):1191-1197

13. Zhu S, Heddam S, Nyarko EK, Hadzima-Nyarko M, Piccolroaz S, Wu S (2019) Modeling daily water temperature for rivers: comparison between adaptive neuro-fuzzy inference systems and artificial neural networks models. Environ Sci Pollut Res 26(1):402-420

14. Yaseen ZM, El-Shafie A, Afan HA, Hameed M, Mohtar WHMW, Hussain A (2016) RBFNN versus FFNN for daily river flow forecasting at Johor River, Malaysia. Neural Comput Appl 27(6):1533-1542

15. Kasiviswanathan K, He J, Sudheer K, Tay J-H (2016) Potential application of wavelet neural network ensemble to forecast streamflow for flood management. J Hydrol 536:161-173

16. Khan UT, He J, Valeo C (2018) River flood prediction using fuzzy neural networks: an investigation on automated network architecture. Water Sci Technol 2017(1):238-247

17. Rigos A, Tsekouras GE, Chatzipavlis A, Velegrakis AF (2016) Modeling beach rotation using a novel Legendre polynomial feedforward neural network trained by nonlinear constrained optimization. In: IFIP international conference on artificial intelligence applications and innovations. Springer, pp 167-179

18. Andrews LC (1992) Special functions of mathematics for engineers. McGraw-Hill, New York

19. Bell WW (2004) Special functions for scientists and engineers. Courier Corporation, North Chelmsford

20. Jiawei H, Kamber M (2011) Data mining: concepts and techniques, (the Morgan Kaufmann series in data management systems), vol 2. Elsevier, Amsterdam

21. Karaboga D (2005) An idea based on honey bee swarm for numerical optimization. In: Technical report-tr06. Erciyes University, Eengineering faculty, computer

22. Kennedy J, Eberhart R (1995) Particle swarm optimization (PSO). In: Proceedings of the IEEE international conference on neural networks, Perth, Australia, pp 1942-1948

23. Zhu G, Kwong S (2010) Gbest-guided artificial bee colony algorithm for numerical function optimization. Appl Math Comput 217(7):3166-3173 
24. Karaboga D, Akay B (2009) A comparative study of artificial bee colony algorithm. Appl Math Comput 214(1):108-132

25. Graf R, Zhu S, Sivakumar B (2019) Forecasting river water temperature time series using a wavelet-neural network hybrid modelling approach. J Hydrol 578:124115. https://doi. org/10.1016/j.jhydrol.2019.124115

26. Bai Y, Chen Z, Xie J, Li C (2016) Daily reservoir inflow forecasting using multiscale deep feature learning with hybrid models. J Hydrol 532:193-206

27. Liu F, Xu F, Yang S (2017) A flood forecasting model based on deep learning algorithm via integrating stacked autoencoders with BP neural network. In: 2017 IEEE third international conference on multimedia big data (BigMM). IEEE, pp 58-61
28. Tsakiri K, Marsellos A, Kapetanakis S (2018) Artificial neural network and multiple linear regression for flood prediction in Mohawk River, New York. Water 10(9):1158

29. Coppola EA Jr, Rana AJ, Poulton MM, Szidarovszky F, Uhl VW (2005) A neural network model for predicting aquifer water level elevations. Groundwater 43(2):231-241

30. Commission C (2013) The critical decade: extreme weather, vol 68. The Climate Commission, Canberra

Publisher's Note Springer Nature remains neutral with regard to jurisdictional claims in published maps and institutional affiliations. 